NBI-HE-99-20

OUTP-99-58P

hep-th/9910195

Revised April 2000

\title{
Bottleneck Surfaces and Worldsheet Geometry of Higher-Curvature Quantum Gravity
}

\author{
Richard J. Szabo \\ The Niels Bohr Institute \\ Blegdamsvej 17, DK-2100 \\ Copenhagen $\varnothing$, Denmark \\ szabo@nbi.dk \\ John F. Wheater \\ Department of Physics - Theoretical Physics \\ University of Oxford \\ 1 Keble Road, Oxford OX1 3NP, U.K. \\ $\mathrm{j}$. wheater1@physics.oxford.ac.uk
}

\begin{abstract}
We describe a simple lattice model of higher-curvature quantum gravity in two dimensions and study the phase structure of the theory as a function of the curvature coupling. It is shown that the ensemble of flat graphs is entropically unstable to the formation of baby universes. In these simplified models the growth in graphs exhibits a branched polymer behaviour in the phase directly before the flattening transition.
\end{abstract}




\section{Introduction}

A longstanding problem of quantum gravity is to determine the effects of the addition of higher-curvature counterterms to the canonical gravity action. This problem is most tractable in two-dimensions, mainly because the theory can be regularized and studied using the discretization approach (see [1] for reviews). In this case, the simplest term of this type that one could add is $\kappa R(g)^{2}$, where $R(g)$ is the scalar curvature of a twodimensional metric $g$ and the coupling constant $\kappa$ is inversely proportional to the square of the worldsheet ultraviolet cutoff. The continuum Euclidean partition function is given by the path integral over all worldsheet metrics

$$
\mathcal{Z}=\int D g \exp \left[-\int_{\Sigma} d^{2} z \sqrt{\operatorname{det} g}\left(\mu+\frac{1}{4 \pi G} R(g)+\kappa R(g)^{2}\right)\right]
$$

where $\mu$ is the cosmological constant and $G$ is the gravitational constant. For $\kappa \rightarrow 0$ the $R^{2}$ term in the action is irrelevant and the system lies in the pure gravitational phase, i.e. that with action consisting of the cosmological and (topological) Einstein terms and with

critical string exponent $\gamma_{\mathrm{str}}=-\frac{1}{2}$ (for a fixed spherical topology). On the other hand, the limit $\kappa \rightarrow \infty$ suppresses large curvature fluctuations of the metric and the statistical ensemble becomes flatter and flatter at short distance scales. At $\kappa=\infty$ only flat surfaces contribute to the partition function.

The problem of whether or not this system undergoes a phase transition between the flat and pure gravitational phases at some finite coupling $\kappa_{c}$ has been studied using quantum Liouville theory in [2] and investigated numerically in [3]. An exact non-perturbative solution has been obtained in [4] for spherical topologies using matrix model techniques and it was shown that $\gamma_{\mathrm{str}}=-\frac{1}{2}$ for all finite values of $\kappa$. The model always reduces at large length scales to a model of pure gravity, i.e. there is no transition to a nonperturbative phase of flat metrics, and only when all surfaces with non-minimal $R(g)^{2}$ are completely forbidden $(\kappa=\infty)$ does $\gamma_{\text {str }}$ change. The key feature of this proof is an appropriate extended model that interpolates between the fixed lattice (or crystalline) flat space model and the pure gravity model [5]. A simplified model which exhibits the same characteristic features has been studied in [6]. In this paper we shall give a simple demonstration of the absence of a phase transition from a random to a flat phase of $R^{2}$ gravity directly in terms of the fractal structure of two-dimensional quantum gravity. This has the advantage of being technically much simpler than the solution given in [4 while at the same time exposing some of the physical and geometrical characteristics of $R^{2}$ gravity.

In section 2 we shall describe how to incorporate the effects of the $R^{2}$ action in twodimensions, using the fact that the value of $\gamma_{\text {str }}$ is controlled by the structure of baby universe formation on the surfaces of the ensemble. This leads to the study of surfaces with branching outgrowths (which we call "bottleneck surfaces") which have a contribution to 
the $R^{2}$ term proportional to the neck thickness. In section 3 we prove that the generating function for the bottleneck surfaces is an analytic function of the curvature coupling $\kappa$, thereby demonstrating the absence of a phase transition as $\kappa$ is continuously varied. Finally, in section 4 we present a model which captures the essential qualitative features of the flattening transition. There we prove that the ensemble of flat graphs is unstable to the formation of baby universe outgrowths, so that the flattening transition can only take place exactly at $\kappa=\infty$. These results show explicitly that no matter how flat the system is at short distance scales, it always destabilizes at long wavelengths into the familiar ensemble of highly fractal baby universes.

\section{Baby Universes and Lattice $R^{2}$ Coupling}

The dynamically triangulated version of pure two-dimensional quantum gravity is given by the partition function

$$
\widehat{Z}(\mu)=\sum_{A} \mathrm{e}^{-\mu A} Z(A)=\sum_{A} \mathrm{e}^{-\mu A} \sum_{\mathrm{T} \in \mathcal{T}_{A}} 1
$$

where $Z(A)$ is the fixed area partition function and $\mathcal{T}_{A}$ is the topological class of triangulations of area $A$ ( $A$ is proportional to the number of triangles of $\mathcal{T}_{A}$ ). A given choice of $\mathrm{T} \in \mathcal{T}_{A}$ corresponds to a particular discretization of the surface. The local intrinsic curvature of a vertex $i \in \mathrm{T}$ of coordination number $q_{i}$ is $R_{i}=2 \pi\left(6-q_{i}\right) / q_{i}$, so that the discretized form of the $R^{2}$ action is given by

$$
\int_{\Sigma} d^{2} z \sqrt{\operatorname{det} g} R(g)^{2} \longrightarrow 4 \pi^{2} \sum_{i \in \mathrm{T}} \frac{\left(q_{i}-6\right)^{2}}{q_{i}}
$$

However, there is a much simpler way to incorporate the effects of the $R^{2}$ term by simply finding an appropriate extended model which interpolates between pure twodimensional quantum gravity and a flat phase of worldsheet metrics. The main idea comes from the fact that the universal constant $\gamma_{\text {str }}$, which describes the nature of the geometry, is related to a surface roughness structure of two-dimensional quantum gravity, the distribution of "minimal bottleneck baby universes" on the ensemble of triangulations [7]. A baby universe of area $A_{0}$ is a small region of the triangulation joined to the bulk solely by a minimal neck, i.e. a loop consisting of only three links. Intuitively, they can be pictured as forming bubblings on the surface. For a triangulation of area $A \rightarrow \infty$ (the continuum limit), the asymptotic behaviour of the fixed area partition function is

$$
Z(A) \simeq A^{\gamma_{\mathrm{str}}-3} \mathrm{e}^{\mu_{0} A}
$$

where $\mu_{0}$ is the critical cosmological constant (so that the series (2.1) converges for all $\left.\mu>\mu_{0}\right)$. From this it can be shown [6] that the distribution $n_{A}\left(A_{0}\right)$ of baby universes is related to the string susceptibility exponent by

$$
n_{A}\left(A_{0}\right) \simeq k A^{3-\gamma_{\mathrm{str}}}\left(A-A_{0}\right)^{\gamma_{\mathrm{str}}-2} A_{0}^{\gamma_{\mathrm{str}}-2}
$$


where here and in the following $k$ denotes a constant which is independent of the areas and is of the order of unity. This result shows that, as $\gamma_{\text {str }}$ increases, the number of baby universes increases as $n_{A}\left(A_{0}\right) / A \simeq k A_{0}^{\gamma_{\text {str }}-2}$. Thus $\gamma_{\text {str }}$ not only measures the fractal structure of the typical surfaces that contribute to the partition function, but also their branching ratio into minimal bottleneck baby universes.

Now consider the situation where the curvature coupling $\kappa$ is very large and the $\int R^{2}$ term is thereby trying to make the surfaces as smooth as possible. A surface of spherical topology has a minimum $\int R^{2}$, denoted $R_{\text {min }}^{2}$, which is independent of its area (basically corresponding to a dual graph consisting of hexagons and 12 pentagons). If we take two such surfaces (not necessarily of the same area) and join them as shown in fig. 1 by cutting out a triangle from each one and stitching them together, then we create a surface with one minimum length bottleneck baby universe and $\int R^{2}=2 R_{\min }^{2}+\Delta$, where $\Delta$ is the contribution to the $\int R^{2}$ term arising from the bottleneck. We can iterate this procedure to produce surfaces with $n$ bottlenecks and

$$
\int R^{2}=R_{\min }^{2}+n\left(R_{\min }^{2}+\Delta\right)
$$

The important point here is that $\int R^{2}$ is not proportional to the area, but only to the number of bottlenecks. On the other hand, most of the surfaces of a given area created in this way are geometrically very different from the single surface with minimum $\int R^{2}$. We can extend this exercise by joining surfaces together with larger, non-minimal necks of length $\ell$. In this case it is clear that the contribution to $\int R^{2}$ can be at most proportional to the length $\ell$ of the bottleneck.

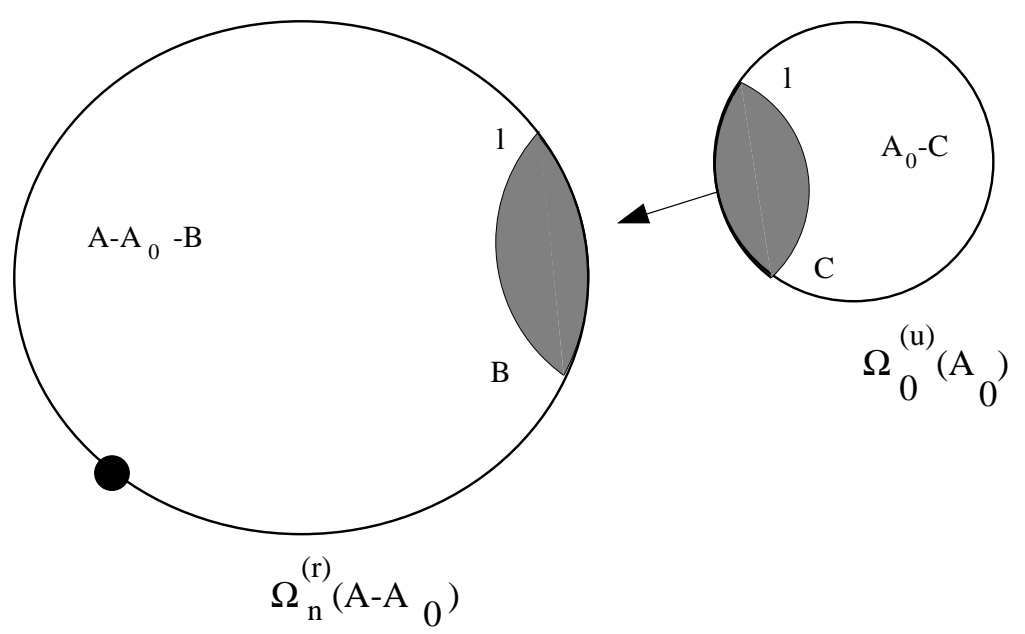

Figure 1: Gluing an unmarked baby graph of area $A_{0}$ (counted by $\Omega_{0}^{(u)}\left(A_{0}\right)$ ) onto a rooted $n$-bottleneck graph of area $A-A_{0}$ (counted by $\Omega_{n}^{(r)}\left(A-A_{0}\right)$ ) along a common deleted disk (shaded areas) to produce a rooted $(n+1)$-bottleneck graph of area $A-B-C$.

To get an idea of how the inclusion of the $R^{2}$ term modifies the distribution functions, it is instructive to consider a simple example. Consider the ensemble of closed rooted trees 
on a fixed lattice. The total number of such trees of length $\ell$ (taken to be the number of links in the tree) is $\mathcal{N}^{(r)}(\ell)$ which behaves asymptotically as (see for example [8])

$$
\mathcal{N}^{(r)}(\ell) \simeq \ell^{\alpha_{0}} \mathrm{e}^{\rho \ell}
$$

where $\alpha_{0}$ is a universal constant which is independent of the particular type of lattice, while $\rho>0$ is a non-universal constant. For a given tree of length $\ell$ there are generically $\ell$ different ways of drawing the tree on the lattice. We introduce an extra fugacity factor $\mathrm{e}^{-\kappa \ell}$ for each loop, which is the analog of the curvature term $\int R^{2}$. The total number of closed rooted trees on a fixed lattice of area $A$ is then

$$
\mathcal{W}(A, \kappa)=\sum_{\ell} \ell \mathcal{N}^{(r)}(\ell) \mathrm{e}^{-\kappa \ell}
$$

For large areas $A \rightarrow \infty$, we can replace the sum in (2.7) by an integral over $\ell \in[0, \infty)$ and suppress the trees which grow too large $\left(\ell^{2} \geq A\right)$ by an exponential damping factor $\mathrm{e}^{-\ell^{2} / A}$. Thus asymptotically we have

$$
\mathcal{W}(A, \kappa) \simeq \int_{0}^{\infty} d \ell \ell \mathcal{N}^{(r)}(\ell) \mathrm{e}^{-\kappa \ell} \mathrm{e}^{-\ell^{2} / A} \simeq k A^{\alpha_{0}+3 / 2} \mathrm{e}^{(\rho-\kappa)^{2} A / 4}
$$

We see that the only effect of the fugacity coupling is to renormalize the entropy parameter as $\rho \rightarrow \rho-\kappa$. The distribution function (2.8) is analytic in $\kappa$ and the inclusion of a fugacity factor for the loops is irrelevant from this point of view. In particular, we may adjust the coupling so that $\kappa=\rho$, in which case $\mathcal{W}(A, \rho)$ has a power law growth with the area. Thus the non-universal tree growth has no effect on the critical behaviour of the corresponding grand canonical ensemble quantities either. Note that for a self-avoiding random walk on

a regular square lattice, we have $\alpha_{0}=-\frac{3}{2}$ (see for example [8]), and $\mathcal{W}(A, \kappa)$ contains a purely exponential growth with the area $A$ of the lattice. By tuning to the point $\kappa=\rho$, the distribution in this case is independent of the size of the lattice.

\section{Analytic Structure of the Bottleneck Graph Generating Function}

We will now write down an expression for the partition function of bottleneck surfaces and argue that it is an analytic function of the $R^{2}$ coupling $\kappa$. We work at fixed spherical topology and consider the generating function for the ensemble of rooted bottleneck graphs which is given by

$$
\widehat{\Omega}^{(r)}\left(\mu, \kappa ; z,\left\{\alpha_{m}\right\}\right)=\sum_{A} \mathrm{e}^{-\mu A} \sum_{n} z^{n} \Omega_{n}^{(r)}\left(A, \kappa ;\left\{\alpha_{m}\right\}\right)=\sum_{A} \mathrm{e}^{-\mu A} \sum_{n} z^{n} \sum_{\mathrm{B} \in \mathcal{B}_{A}^{n}} w_{\mathrm{B}}
$$

where $\mathcal{B}_{A}^{n}$ denotes the set of all $n$-bottleneck surfaces of area $A$. The local weight of a graph $\mathrm{B} \in \mathcal{B}_{A}^{n}$ is given by

$$
w_{\mathrm{B}}=\prod_{b \in \mathrm{B}} \alpha_{q_{b}-1} \mathrm{e}^{-\kappa \ell_{b}}
$$


where $b$ are the bottleneck nodes of $\mathrm{B}$ of branching number $q_{b}$ and neck thickness $\ell_{b}$, and $\alpha_{m}$ are arbitrary weights associated with the nodes. The number $\Omega_{n}^{(r)}(A)$ of rooted spherical graphs of area $A$ with $n$ bottlenecks (where the area of each triangle is taken to be unity) can be constructed inductively as follows. For $n=0$ this number is related to the number $\Omega_{0}^{(u)}(A) \equiv Z(A)$ of unmarked spherical graphs as $\Omega_{0}^{(r)}(A)=A \Omega_{0}^{(u)}(A)$, since there are $A$ possible triangles to mark in an unmarked graph. To construct a rooted graph with $n+1$ bottlenecks, we cut out a disk of area $B$ and perimeter $\ell$ from a rooted graph with $n$ bottlenecks, and a disk of area $C$ with the same boundary length $\ell$ from a 0-neck unmarked graph (fig. 1). Gluing the two cut graphs together along the common perimeter of the deleted disks yields a rooted graph with $n+1$ bottlenecks. In doing so, one must be careful of the degeneracies which occur in this inductive cutting procedure. Consider two spherical (0-neck) graphs which are identical to each other except for their triangulations inside two disks, of areas $B_{0}$ and $C_{0}$ and with identical boundary length $\ell$, on each respective graph. These two inequivalent graphs, when glued onto other lattices to produce bottleneck graphs, yield the same set of surfaces.

We shall first consider the sub-ensemble of linear chain bottleneck graphs. We introduce the disk amplitude $Z_{1}(\ell ; B)$ which is defined to be the number of surfaces of area $B$ and one boundary of length $\ell$. If we join two surfaces of areas $B_{0}$ and $B-B_{0}>B_{0}$, each having a single boundary of length $\ell$, along their common boundaries, then we obtain a closed surface of area $B$ with a marked loop of length $\ell$ partitioning it into two parts of areas $B_{0}$ and $B-B_{0}$. Since the boundary has $\ell$ links, there are generically $\ell$ different ways of joining the two surfaces to obtain distinct final surfaces (provided that $B_{0}$ and $B-B_{0}$ are large enough). Moreover, any surface of area $B$ with a marked loop of length $\ell$ partitioning it into two parts of areas $B_{0}$ and $B-B_{0}$ can be uniquely constructed in this way. Therefore, for large $B$ and $B_{0}$, there are

$$
G_{1}\left(B, B_{0} ; \ell\right) \simeq \ell Z_{1}\left(\ell ; B_{0}\right) Z_{1}\left(\ell ; B-B_{0}\right)
$$

closed surfaces of area $B$ with a marked loop of length $\ell$ that partitions the surface into two parts of areas $B_{0}$ and $B-B_{0}$. The asymptotic large area behaviour of the disk amplitude is 9

$$
Z_{1}(\ell ; B) \simeq B^{\gamma_{\mathrm{str}}-2} \mathrm{e}^{\mu_{0} B} \ell^{-\gamma_{\mathrm{str}}-1} \mathrm{e}^{\rho \ell}
$$

where $\rho>0$ is as in (2.6). This amplitude therefore increases with $\ell$ for small $\ell$, and for large perimeter loops $\left(\ell^{2} \geq B\right), Z_{1}(\ell ; B)$ is exponentially damped by worldsheet finite size cutoff factors like $\mathrm{e}^{-\ell^{2} / B}[9]$.

We shall also use the annulus amplitude $Z_{2}\left(\ell_{1}, \ell_{2} ; B\right)$ which is the number of surfaces of area $B$ with two holes of boundary lengths $\ell_{1}$ and $\ell_{2}$. Its large area asymptotic behaviour is

$$
Z_{2}\left(\ell_{1}, \ell_{2} ; B\right) \simeq B^{\gamma_{s t r}-1} \mathrm{e}^{\mu_{0} B}\left(\ell_{1}+\ell_{2}\right)^{-\gamma_{\mathrm{str}}-1} \mathrm{e}^{\rho\left(\ell_{1}+\ell_{2}\right)}
$$

and for large loops it is suppressed by the worldsheet infrared cutoff $\mathrm{e}^{-\left(\ell_{1}+\ell_{2}\right)^{2} / B}$ [9]. Then 
there are

$$
G_{2}\left(B, B_{1}, B_{2} ; \ell_{1}, \ell_{2}\right)=\ell_{1} \ell_{2} Z_{1}\left(\ell_{1} ; B_{1}\right) Z_{1}\left(\ell_{2} ; B_{2}\right) Z_{2}\left(\ell_{1}, \ell_{2} ; B-B_{1}-B_{2}\right)
$$

distinct surfaces of area $B$ with two non-intersecting loops of lengths $\ell_{1}$ and $\ell_{2}$ enclosing areas $B_{1}$ and $B_{2}$, respectively. Neglecting the graphs counted by $Z_{1}(\ell ; B)$ and $Z_{2}\left(\ell_{1}, \ell_{2} ; B\right)$ which themselves have bottlenecks, i.e. assuming that (3.4) and (3.5) correctly count the disk and annulus graphs with no bottlenecks of lengths smaller than $\ell$ and $\ell_{1}, \ell_{2}$, an $n$-neck linear chain can then be constructed by gluing together $n-1$ annulus graphs in between two disk graphs. We assume that there is a one-to-one correspondence between the latter configurations of graphs and those obtained by slicing an $n$-bottleneck graph along each of its necks. These assumptions will be sufficient to determine the analytic properties of the full generating function.

We may now write down an expression for the number $\mathcal{L}_{n}^{(r)}\left(A ; \ell_{1}, \ldots, \ell_{n}\right)$ of rooted linear graphs of total area $A$ and with $n$ bottlenecks of lengths $\ell_{1}, \ldots, \ell_{n}$. In the continuum limit, we can replace sums over areas and bottleneck loop lengths by integrals. The lower limits of integration for the perimeter integrals are 3 links (the minimal bottleneck size), while those of the area integrals are 1 triangle. As all area integrations are ultraviolet finite, there is no danger in continuing their limits down to $A_{i}=0$. Incorporating the smooth infrared cutoffs on the lengths as described above and the loop fugacity factor $\mathrm{e}^{-2 \kappa \ell_{i}}$ for each neck, this yields the combinatorial identity

$$
\begin{aligned}
\mathcal{L}_{n}^{(r)}\left(A, \kappa ; \ell_{1}, \ldots, \ell_{n}\right)= & \left(\prod_{i=1}^{n+1} \int_{0}^{\infty} d A_{i}\right) \delta\left(A-\sum_{i} A_{i}\right) A_{1} \ell_{1} Z_{1}\left(\ell_{1} ; A_{1}\right) \mathrm{e}^{-2 \kappa \ell_{1}} \mathrm{e}^{-\ell_{1}^{2} / A_{1}} \\
& \times Z_{1}\left(\ell_{n} ; A_{n+1}\right) \mathrm{e}^{-\ell_{n}^{2} / A_{n+1}} \\
& \times \prod_{i=1}^{n-1} \ell_{i+1} Z_{2}\left(\ell_{i}, \ell_{i+1} ; A_{i+1}\right) \mathrm{e}^{-2 \kappa \ell_{i+1}} \mathrm{e}^{-\left(\ell_{i}+\ell_{i+1}\right)^{2} / A_{i}}
\end{aligned}
$$

It is convenient to analyse this relation in the grand canonical ensemble. For this, we define the Laplace transforms of the loop amplitudes by

$$
\begin{aligned}
\widehat{Z}_{1}(\ell ; \mu) & \equiv \int_{0}^{\infty} d B \mathrm{e}^{-\mu B} Z_{1}(\ell ; B) \mathrm{e}^{-\ell^{2} / B} \\
& \simeq \frac{\left(\sqrt{\mu-\mu_{0}}\right)^{1-\gamma_{\mathrm{str}}}}{\ell^{2}} \mathrm{e}^{\rho \ell} K_{1-\gamma_{\mathrm{str}}}\left(2 \sqrt{\mu-\mu_{0}} \ell\right) \\
\widehat{Z}_{2}\left(\ell_{1}, \ell_{2} ; \mu\right) & \equiv \int_{0}^{\infty} d B \mathrm{e}^{-\mu B} Z_{2}\left(\ell_{1}, \ell_{2} ; B\right) \mathrm{e}^{-\left(\ell_{1}+\ell_{2}\right)^{2} / B} \\
& \simeq \frac{\left(\sqrt{\mu-\mu_{0}}\right)^{-\gamma_{\mathrm{str}}}}{\ell_{1}+\ell_{2}} \mathrm{e}^{\rho\left(\ell_{1}+\ell_{2}\right)} K_{-\gamma_{\mathrm{str}}}\left(2 \sqrt{\mu-\mu_{0}}\left(\ell_{1}+\ell_{2}\right)\right)
\end{aligned}
$$

where we have used (3.4) and (3.5), and $K_{\nu}$ is the irregular modified Bessel function of order $\nu$. The corresponding expression in the grand canonical ensemble is thus

$\widehat{\mathcal{L}}_{n}^{(r)}\left(\mu, \kappa ; \ell_{1}, \ldots, \ell_{n}\right) \equiv \int_{0}^{\infty} d A \mathrm{e}^{-\mu A} \mathcal{L}_{n}^{(r)}\left(A, \kappa ; \ell_{1}, \ldots, \ell_{n}\right)$ 


$$
\begin{aligned}
= & \frac{\left(\sqrt{\mu-\mu_{0}}\right)^{1-(n+1) \gamma_{\mathrm{str}}}}{\ell_{1} \ell_{n}} K_{-\gamma_{\mathrm{str}}}\left(2 \sqrt{\mu-\mu_{0}} \ell_{1}\right) K_{1-\gamma_{\mathrm{str}}}\left(2 \sqrt{\mu-\mu_{0}} \ell_{n}\right) \\
& \times \mathrm{e}^{2(\rho-\kappa) \sum_{i} \ell_{i}} \prod_{i=1}^{n-1} \frac{\ell_{i}}{\ell_{i}+\ell_{i+1}} K_{-\gamma_{\mathrm{str}}}\left(2 \sqrt{\mu-\mu_{0}}\left(\ell_{i}+\ell_{i+1}\right)\right)
\end{aligned}
$$

We are now interested in the analytic properties of the total distribution of linear $n$-bottleneck graphs

$$
\widehat{\mathcal{L}}_{n}^{(r)}(\mu, \kappa) \equiv\left(\prod_{i=1}^{n} \int_{3}^{\infty} d \ell_{i}\right) \widehat{\mathcal{L}}_{n}^{(r)}\left(\mu, \kappa ; \ell_{1}, \ldots, \ell_{n}\right)
$$

as a function of $\kappa \in \mathbb{R}^{+}$. Because of the ultraviolet cutoff on the length integrations, possible singularities in (3.10) would come only from the behaviour of the integral as $\ell_{i} \rightarrow \infty$. For our purposes we may therefore approximate the modified Bessel functions in (3.9) by their asymptotic behaviours $K_{\nu}(z) \sim z^{-1 / 2} \mathrm{e}^{-z}$ for $|z| \rightarrow \infty$ and consider the function

$$
\begin{aligned}
\widehat{\mathcal{L}}_{n}^{(r)}(\mu, \kappa) \simeq & \frac{\left(\sqrt{\mu-\mu_{0}}\right)^{-\frac{1}{2}(n-1)-(n+1) \gamma_{\mathrm{str}}}}{(\sqrt{2})^{n+1}} \\
& \times\left(\prod_{i=1}^{n} \int_{3}^{\infty} d \ell_{i} \mathrm{e}^{2\left(\rho-\kappa-2 \sqrt{\mu-\mu_{0}}\right) \ell_{i}}\right) \frac{1}{\ell_{1}^{3 / 2} \ell_{n}^{3 / 2}} \prod_{i=1}^{n-1} \frac{\ell_{i}}{\left(\ell_{i}+\ell_{i+1}\right)^{3 / 2}}
\end{aligned}
$$

In order that (3.11) be generically convergent, we must be in the phase with $\mu \geq \mu_{c}$, where

$$
\mu_{c}=\mu_{0}+\frac{(\rho-\kappa)^{2}}{4}
$$

At $\mu=\mu_{c}$ there is a phase transition, but the critical behaviour is just that of the usual continuum limit of the discretized surface model. This will follow from the fact that both (3.11) and (3.12) are analytic functions of $\kappa$, and the usual critical point $\mu_{c}=\mu_{0}$ can be reached by tuning the $R^{2}$ coupling to the value $\kappa=\rho$. We now focus on the analytic properties of $\widehat{\mathcal{L}}_{n}^{(r)}(\mu, \kappa)$ assuming that $\mu \geq \mu_{c}$. It can be uniformly bounded by using the inequality $\ell_{i}+\ell_{i+1} \geq \sqrt{2 \ell_{i} \ell_{i+1}}$ to write down an upper bound on the integrations on the right-hand side of (3.11) which can be evaluated in terms of the incomplete gammafunction $\Gamma(\alpha, x)=\int_{x}^{\infty} d t t^{\alpha-1} \mathrm{e}^{-t}$ to give a bound on the derivatives of $\widehat{\mathcal{L}}_{n}^{(r)}(\mu, \kappa)$ with respect to $\kappa$,

$$
\begin{aligned}
\left|\frac{\partial^{k} \widehat{\mathcal{L}}_{n}^{(r)}(\mu, \kappa)}{\partial \kappa^{k}}\right| \leq & \frac{\left(\sqrt{\mu-\mu_{0}}\right)^{-\frac{1}{2}(n-1)-(n+1) \gamma_{\text {str }}}}{2^{(7 n-11) / 4-k(n+1)}}\left(2 \sqrt{\mu-\mu_{0}}+\kappa-\rho\right)^{-(n-5) / 2+k n} \\
& \times \Gamma\left(-\frac{1}{4}+k, 6\left(2 \sqrt{\mu-\mu_{0}}+\kappa-\rho\right)\right) \Gamma\left(-\frac{5}{4}+k, 6\left(2 \sqrt{\mu-\mu_{0}}+\kappa-\rho\right)\right) \\
& \times \Gamma\left(\frac{1}{2}+k, 6\left(2 \sqrt{\mu-\mu_{0}}+\kappa-\rho\right)\right)^{n-2}
\end{aligned}
$$

for $k \geq 0$.

For non-integer or negative $\alpha, \Gamma(\alpha, x)$ is a multi-valued function of $x$ with a branch cut along the negative real $x$-axis. In the phase with $\mu>\mu_{c}$, the linear $n$-bottleneck 
distribution is therefore uniformly bounded by an analytic function of $2 \sqrt{\mu-\mu_{0}}+\kappa-\rho$. In terms of the generating function for the linear bottleneck graph ensemble, defined as a formal power series

$$
\widehat{\mathcal{L}}^{(r)}(\mu, \kappa ; z)=\sum_{n=0}^{\infty} z^{n} \widehat{\mathcal{L}}_{n}^{(r)}(\mu, \kappa)
$$

in a variable $z$, this bound reads

$$
\begin{aligned}
\left|\frac{\partial^{k} \widehat{\mathcal{L}}^{(r)}(\mu, \kappa ; z)}{\partial \kappa^{k}}\right| \leq & \frac{\Gamma\left(-\frac{1}{4}+k, 6\left(2 \sqrt{\mu-\mu_{0}}+\kappa-\rho\right)\right) \Gamma\left(-\frac{5}{4}+k, 6\left(2 \sqrt{\mu-\mu_{0}}+\kappa-\rho\right)\right)}{\Gamma\left(\frac{1}{2}+k, 6\left(2 \sqrt{\mu-\mu_{0}}+\kappa-\rho\right)\right)^{2}} \\
& \times 2^{2 k+3}\left(\sqrt{\mu-\mu_{0}}\right)^{\frac{1}{2}-\gamma_{\text {str }}}\left(2 \sqrt{\mu-\mu_{0}}+\kappa-\rho\right)^{5 / 2} \\
& \times\left[2^{k+1 / 4}-4 z\left(\sqrt{\mu-\mu_{0}}\right)^{-\frac{1}{2}-\gamma_{\text {str }}}\left(2 \sqrt{\mu-\mu_{0}}+\kappa-\rho\right)^{-1 / 2+k}\right. \\
& \left.\times \Gamma\left(\frac{1}{2}+k, 6\left(2 \sqrt{\mu-\mu_{0}}+\kappa-\rho\right)\right)\right]^{-1}
\end{aligned}
$$

Being uniformly bounded by an analytic function, the generating function cannot have any singularities as $\kappa>\rho-2 \sqrt{\mu-\mu_{0}}$ is varied and it is analytic in the variable $2 \sqrt{\mu-\mu_{0}}+$ $\kappa-\rho$. Furthermore, since the critical cosmological constant (3.12) is itself an analytic function of $\kappa$, there are no singularities as $\kappa$ is varied throughout its range. Therefore, the linear bottleneck generating function is an analytic function of $\kappa \in \mathbb{R}^{+}$. There are no phase transitions within the ensemble of linear bottleneck graphs as one continuously varies the $R^{2}$ coupling constant.

We now consider the full bottleneck graph ensemble with generating function (3.1). To study a bottleneck distribution with arbitrary branchings, we shall need to use the genus $0 n$-loop amplitude $Z_{n}\left(\ell_{1}, \ldots, \ell_{n} ; B\right)$. We can determine its approximate asymptotic behaviour as follows. For each additional loop that is drawn on a graph there is an extra combinatorial area factor giving an overall entropy $B^{n} Z(B)$ for locating the centers of the loops on the closed surface. Furthermore, each loop on the surface contributes an exponential length growth $\mathrm{e}^{\rho \ell_{i}}$, and the smooth infrared cutoff on large perimeter loops is $\mathrm{e}^{-\left(\sum_{i} \ell_{i}\right)^{2} / B}$. The crucial point, however, is that the $n$-loop amplitude depends only on the sum of the loop lengths [9]. The natural ansatz is then

$$
Z_{n}\left(\ell_{1}, \ldots, \ell_{n} ; B\right) \simeq B^{\gamma_{\mathrm{str}}-3+n} \mathrm{e}^{\mu_{0} B}\left(\sum_{i=1}^{n} \ell_{i}\right)^{-\gamma_{\mathrm{str}}-1} \mathrm{e}^{\rho \sum_{i} \ell_{i}} \mathrm{e}^{-\left(\sum_{i} \ell_{i}\right)^{2} / B}
$$

We note that this ansatz only dictates the large area and loop length dependence of the amplitude, but not its coefficient which depends on $n$.

To establish (3.16), we use induction on $n$ and the consistency condition

$$
Z_{n-1}\left(\ell_{1}, \ldots, \ell_{n-1} ; B\right)=\int_{1}^{B} d B_{0} \int_{3}^{\infty} d \ell Z_{n}\left(\ell_{1}, \ldots, \ell_{n-1}, \ell ; B_{0}\right) Z_{1}\left(\ell ; B-B_{0}\right)
$$

which represents the combinatorics of gluing together disk amplitudes and $n$-loop amplitudes to generate $(n-1)$-loop graphs. Using the ansatz (3.16) and changing variables in 
the area integral to $x=B_{0} /\left(B-B_{0}\right)$, the right-hand side of (3.17) becomes

$$
\begin{aligned}
& B^{2 \gamma_{\mathrm{str}}-4+n} \mathrm{e}^{\mu_{0} B} \mathrm{e}^{\rho L_{n-1}} \mathrm{e}^{-L_{n-1}^{2} / B} \int_{3}^{\infty} d \ell \ell^{-\gamma_{\mathrm{str}}-1}\left(\ell+L_{n-1}\right)^{-\gamma_{\mathrm{str}}-1} \mathrm{e}^{2 \rho \ell-2 \ell\left(\ell+L_{n-1}\right) / B} \\
& \quad \times \int_{-1}^{\infty} d x x^{\gamma_{\mathrm{str}}-3+n}(x+1)^{-2 \gamma_{\mathrm{str}}+3-n} \mathrm{e}^{-\ell^{2} x / B-\left(\ell+L_{n-1}\right)^{2} / B x}
\end{aligned}
$$

where $L_{n-1}=\sum_{i=1}^{n-1} \ell_{i}$. For $B \rightarrow \infty$ we may approximate the integral over $x$ in (3.18) by its asymptotic behaviour which integrates to the modified Bessel function

$$
2\left(\frac{\ell+L_{n-1}}{\ell}\right)^{\gamma_{\mathrm{str}}-1} K_{\gamma_{\mathrm{str}}-1}\left(2 \ell\left(\ell+L_{n-1}\right) / B\right)
$$

From the asymptotic behaviour $K_{\nu}(x) \simeq 2^{\nu-1} \Gamma(\nu) x^{-\nu}+\ldots$ for $x \rightarrow 0$ and $\nu \neq 0$, it follows that the large-area behaviour of $(3.18)$ is thus

$$
B^{3 \gamma_{\mathrm{str}}-5+n} \mathrm{e}^{\mu_{0} B} \mathrm{e}^{\rho L_{n-1}} \mathrm{e}^{-L_{n-1}^{2} / B} \int_{3}^{\infty} d \ell \ell^{-\gamma_{\mathrm{str}}+1}\left(\ell+L_{n-1}\right)^{-\gamma_{\mathrm{str}}-1} \mathrm{e}^{2 \rho \ell-2 \ell\left(\ell+L_{n-1}\right) / B}
$$

Within the present approximations the loop integral (3.20) may be evaluated using the saddle-point approximation. The stationary condition is

$$
2\left(\rho-\frac{L_{n-1}}{B}\right) \ell\left(\ell+L_{n-1}\right)-\frac{4 \ell^{2}\left(\ell+L_{n-1}\right)}{B}-\left(\gamma_{\mathrm{str}}+1\right) \ell-\left(\gamma_{\mathrm{str}}-1\right)\left(\ell+L_{n-1}\right)=0
$$

Solving the cubic equation (3.21) and evaluating the one-loop fluctuation integral corresponding to (3.20) for the root which is positive and regular at $L_{n-1}=0$, we find in the limit $B \rightarrow \infty$ that (3.20) is proportional to the left-hand side of (3.17) as given by (3.16). Taking the Laplace transform of the expression (3.16), we find the corresponding amplitude in the grand canonical ensemble,

$$
\begin{aligned}
\widehat{Z}_{n}\left(\ell_{1}, \ldots, \ell_{n} ; \mu\right) & \simeq\left(\sqrt{\mu-\mu_{0}}\right)^{2-n-\gamma_{\text {str }}}\left(\sum_{i=1}^{n} \ell_{i}\right)^{n-3} \mathrm{e}^{\rho \sum_{i} \ell_{i}} K_{2-n-\gamma_{\mathrm{str}}}\left(2 \sqrt{\mu-\mu_{0}} \sum_{i} \ell_{i}\right) \\
& \sim\left(\sqrt{\mu-\mu_{0}}\right)^{\frac{3}{2}-n-\gamma_{\mathrm{str}}}\left(\sum_{i=1}^{n} \ell_{i}\right)^{n-\frac{7}{2}} \mathrm{e}^{\left(\rho-2 \sqrt{\mu-\mu_{0}}\right) \sum_{i} \ell_{i}} \quad \text { for } \ell_{i} \rightarrow \infty
\end{aligned}
$$

With the bottleneck node couplings $\alpha_{m}$ completely arbitrary, the full generating function may be generated by the recursive equation which is represented symbolically in fig. 2. Summing over all configurations shown there gives the identity

$$
\begin{aligned}
& \int_{0}^{\infty} d A_{1} d A_{2} \delta\left(A-A_{1}-A_{2}\right) \int_{3}^{\infty} d \ell \ell \mathrm{e}^{-\kappa \ell} Z_{1}^{(r)}\left(\ell ; A_{1}\right) \Omega\left(\ell, A_{2}, \kappa ; z,\left\{\alpha_{m}\right\}\right) \\
& =z\left[A Z(A)+\sum_{n=1}^{\infty} \alpha_{n}\left(\prod_{i=1}^{n+1} \int_{0}^{\infty} d A_{i}\right) \delta\left(A-\sum_{i} A_{i}\right)\left(\prod_{i=1}^{n} \int_{3}^{\infty} d \ell_{i} \ell_{i} \mathrm{e}^{-\kappa \ell_{i}}\right)\right. \\
& \left.\quad \times Z_{n}^{(r)}\left(\ell_{1}, \ldots, \ell_{n} ; A_{1}\right) \prod_{i=1}^{n} \Omega\left(\ell_{i}, A_{i+1}, \kappa ; z,\left\{\alpha_{m}\right\}\right)\right]
\end{aligned}
$$


where $Z_{n}^{(r)}\left(\ell_{1}, \ldots, \ell_{n} ; A\right)=A Z_{n}\left(\ell_{1}, \ldots, \ell_{n} ; A\right)$ is the marked $n$-loop amplitude and we have introduced the generating function $\Omega\left(\ell, A, \kappa ; z,\left\{\alpha_{m}\right\}\right)$ for bottleneck surfaces of area $A$ and with a single deleted disk of boundary length $\ell$. The left-hand side of (3.23) is the desired quantity $\Omega^{(r)}\left(A, \kappa ; z,\left\{\alpha_{m}\right\}\right)$ and from (3.22) it follows that the corresponding relationship in the grand canonical ensemble is

$$
\begin{aligned}
\widehat{\Omega}^{(r)}\left(\mu, \kappa ; z,\left\{\alpha_{m}\right\}\right) \equiv & \int_{3}^{\infty} d \ell \ell \mathrm{e}^{(\rho-\kappa) \ell} K_{-\gamma_{\mathrm{str}}}\left(2 \sqrt{\mu-\mu_{0}} \ell\right) \widehat{\Omega}\left(\ell, \mu, \kappa ; z,\left\{\alpha_{m}\right\}\right) \\
= & z\left[\left(\mu-\mu_{0}\right)^{1-\gamma_{\mathrm{str}}}+\sum_{n=1}^{\infty} \alpha_{n}\left(\sqrt{\mu-\mu_{0}}\right)^{1-n-\gamma_{\mathrm{str}}}\right. \\
& \times \prod_{i=1}^{n} \int_{3}^{\infty} d \ell_{i} \ell_{i} \mathrm{e}^{(\rho-\kappa) \ell_{i}} \widehat{\Omega}\left(\ell_{i}, \mu, \kappa ; z,\left\{\alpha_{m}\right\}\right) \\
& \left.\times\left(\sum_{i=1}^{n} \ell_{i}\right)^{n-2} K_{1-n-\gamma_{\mathrm{str}}}\left(2 \sqrt{\mu-\mu_{0}} \sum_{i} \ell_{i}\right)\right]
\end{aligned}
$$

This iterative relation defines a complicated integral equation for $\widehat{\Omega}\left(\ell, \mu, \kappa ; z,\left\{\alpha_{m}\right\}\right)$ whose solution gives the generating function (3.1). The complexity of the identity (3.24) makes an explicit solution or even an analyticity analysis intractable. However, one may argue that the solution to (3.24) is an analytic function of $\kappa$ as follows. A generic branching distribution function for the bottleneck ensemble will always involve products of functions of the form (3.22). From the inequalities

$$
\left(\sum_{i=1}^{n} \ell_{i}\right)^{n-\frac{7}{2}} \leq\left\{\begin{array}{cc}
n^{\left(n-\frac{7}{2}\right) / n} \prod_{i} \ell_{i}^{\left(n-\frac{7}{2}\right) / n} & n<4 \\
n^{n-\frac{7}{2}} \max _{i} \ell_{i}^{n-\frac{7}{2}} & , \quad n \geq 4
\end{array}\right.
$$

it follows that a general bottleneck function can always be bounded from above by a function which is given by a product of incomplete gamma-functions and other elementary functions. In the phase $\mu>\mu_{c}$, the $n$-neck distribution functions are analytic in $\rho$. The grand canonical distribution function $\widehat{\Omega}^{(r)}(\mu, \kappa ; z)$ is therefore expected to be an analytic function of $\kappa \in \mathbb{R}^{+}$. Note that the recursive definition (3.24) is reminescent of that for the generating function of a branched polymer ensemble [10]. In the next section we shall consider a slight simplification of the model defined by (3.24) which is amenable to explicit analysis and thereby demonstrate that this similarity is not a coincidence.

\section{A Model for the Flattening Transition}

We shall now present a model for the transition between flat and random graphs which captures the essential qualitative features of the flattening transition. We will consider two simplifications of the full bottleneck graph generating function. First, we start with 


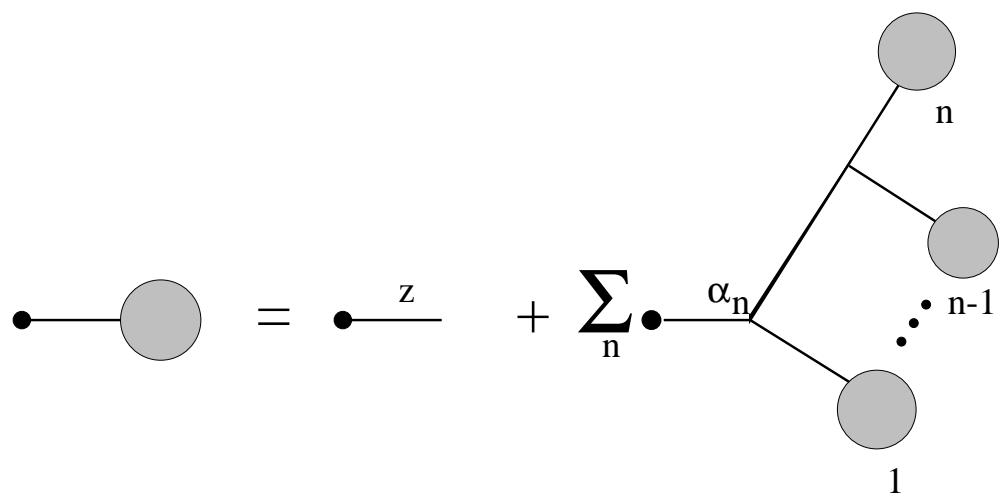

Figure 2: Schematic representation of the iterative definition of the bottleneck surface generating function. The shaded circles represent the loop functions $\Omega\left(\ell, A, \kappa ; z,\left\{\alpha_{m}\right\}\right)$ while the lines represent 0-neck surfaces. Each attachment is done by gluing the surfaces together along a common deleted disk.

the ensemble of regular square lattices, f which in the schematic representation of fig. 2 are represented by the rooted lines. Let $\mathcal{Z}_{\ell}^{(0)}(A)$ be the number of such flat graphs of area $A$ and with a single square loop of side $\ell$ drawn on them (the 0-bottleneck partition function). We are interested in the generating function $\mathcal{Z}_{\ell}(A)$ for the ensemble of bottleneck surfaces of area $A$ with a single loop of side $\ell$ drawn on them, which is depicted by the shaded circles in fig. 2. It is constructed recursively as described at the end of the previous section. We start with a flat base lattice of area $A_{0}$ and put an outgrowth of bottleneck graphs with a square loop of side $n$ on it. There are $A_{0}$ possible ways to do this and the area of the new graph is $A_{0}-n^{2}$. However, the effective area remaining to insert a second outgrowth of bottleneck surfaces of loop perimeter $4 n$ is $A_{0}-4 n^{2}$ because of the excluded area effect. Each such outgrowth is attached to the base graph with a fugacity $\mathrm{e}^{-\kappa w(n)}$, where $w(n)$ is a function which satisfies $w(1)=1$ and $w^{\prime}(n) \geq 0$, but which does not grow as fast as $n^{2}$.

This procedure can be used to build the full generating function $\mathcal{Z}_{\ell}(A)$ by summing over all possible ways of attaching outgrowths to the base graph. Dividing by the permutation symmetry factor for a $k$-branch surface, we have

$$
\begin{aligned}
\mathcal{Z}_{\ell}(A, \kappa)= & \mathcal{Z}_{\ell}^{(0)}(A)+\sum_{A_{0}=\ell^{2}}^{\infty} \mathcal{Z}_{\ell}^{(0)}\left(A_{0}\right) \sum_{n=1}^{\frac{1}{2} \sqrt{A_{0}}} \sum_{k=1}^{A_{0} / 4 n^{2}} \frac{1}{k !} \\
& \times\left[\prod_{i=1}^{k}\left(A_{0}-4(i-1) n^{2}\right) \sum_{A_{i}=n^{2}}^{\infty} \mathcal{Z}_{n}\left(A_{i}, \kappa\right) \mathrm{e}^{-\kappa w(n)}\right] \delta\left(A-A_{0}+k n^{2}-\sum_{i} A_{i}\right)
\end{aligned}
$$

${ }^{*}$ Actually, one must consider square lattices with positive curvature defect insertions to be able to close them on a spherical topology. This modification would not affect the qualitative behaviour described in the following. The model could of course be extended to include nonsquare lattices and arbitrarily shaped bottlenecks. 
It is again convenient to analyse the grand canonical partition function which here is defined by the discrete Laplace transform

$$
\begin{aligned}
\widehat{\mathcal{Z}}_{\ell}(\mu, \kappa) & \equiv \sum_{A=\ell^{2}}^{\infty} \mathrm{e}^{-\mu A} \mathcal{Z}_{\ell}(A, \kappa) \\
& =\widehat{\mathcal{Z}}_{\ell}^{(0)}(\mu)+\sum_{A=\ell^{2}}^{\infty} \sum_{n=1}^{\frac{1}{2} \sqrt{A}} \mathrm{e}^{-\mu A} \mathcal{Z}_{\ell}^{(0)}(A)\left[\left(1+4 n^{2} \mathrm{e}^{\mu n^{2}-\kappa w(n)} \widehat{\mathcal{Z}}_{n}(\mu, \kappa)\right)^{A / 4 n^{2}}-1\right]
\end{aligned}
$$

where we have used the binomial theorem to do the sum over $k$ from (4.1). Upon carefully interchanging the sums over $n$ and $A$ in (4.2) we arrive at

$$
\widehat{\mathcal{Z}}_{\ell}(\mu, \kappa)=\widehat{\mathcal{H}}_{\ell}^{(0)}(\mu)+\sum_{n=1}^{\ell-1} \widehat{\mathcal{Z}}_{\ell}^{(0)}\left(\bar{\mu}_{n}\right)+\sum_{n=\ell}^{\infty} \widehat{\mathcal{Z}}_{n}^{(0)}\left(\bar{\mu}_{n}\right)
$$

where we have introduced the function

$$
\widehat{\mathcal{H}}_{\ell}^{(0)}(\mu)=\widehat{\mathcal{Z}}_{\ell}^{(0)}(\mu)-\frac{1}{2} \sum_{A=\ell^{2}}^{\infty} \sqrt{A} \mathrm{e}^{-\mu A} \mathcal{Z}_{\ell}^{(0)}(A),
$$

and the rescaled cosmological constants

$$
\bar{\mu}_{n}(\mu, \kappa)=\mu-\frac{1}{4 n^{2}} \log \left(1+4 n^{2} \mathrm{e}^{\mu n^{2}-\kappa w(n)} \widehat{\mathcal{Z}}_{n}(\mu, \kappa)\right) .
$$

Note that the grand canonical base graph generating function behaves asymptotically for large loops as

$$
\widehat{\mathcal{Z}}_{\ell}^{(0)}(\mu)=\mathrm{e}^{-\mu \ell^{2}} g_{\ell}(\mu)
$$

where $g_{\ell}(\mu)<g_{1}(\mu)$ and $g_{1}(\mu)$ converges for all positive $\mu$ and diverges at $\mu=0$. It follows that the sums in (4.3) converge provided $\bar{\mu}_{n}(\mu, \kappa)>0$.

We will now analyse the analytic properties of the solution of the infinite-term recursion relation (4.3) as a function of $\mu$ and $\kappa$. From (4.2) it follows that

$$
\widehat{\mathcal{Z}}_{\ell}(\mu, \kappa=\infty)=\widehat{\mathcal{Z}}_{\ell}^{(0)}(\mu)
$$

so that the suppression of curvature fluctuations via an infinite $R^{2}$-coupling leaves only flat (square lattice) graphs. In this flat phase, the partition function diverges only at the critical point $\mu_{c}^{(0)}=0$ and formally the string susceptibility exponent is $\gamma_{\mathrm{str}}^{(0)}=2$. For $\kappa<\infty$, it follows immediately from (4.3) that the partition function $\widehat{\mathcal{Z}}_{\ell}(\mu, \kappa)$ determines a different universality class than the flat phase. To see this, we suppose that $\widehat{\mathcal{Z}}_{\ell}(\mu, \kappa)$ has the same critical behaviour as $\widehat{\mathcal{Z}}_{\ell}^{(0)}(\mu)$, i.e. that $\widehat{\mathcal{Z}}_{\ell}(\mu, \kappa)$ diverges at some critical point $\mu=\mu_{c}$. If this were the case then the smallest of the $\bar{\mu}_{n}(\mu, \kappa)$ in (4.5) would become zero at some $\mu=\mu_{c}^{\prime}>\mu_{c}$, and so from (4.3) and (4.6) the partition function $\widehat{\mathcal{Z}}_{\ell}(\mu, \kappa)$ diverges before its critical point is reached. Thus a critical behaviour at any $\kappa<\infty$ whereby 
$\widehat{\mathcal{Z}}_{\ell}(\mu, \kappa)$ itself diverges is inconsistent. It is only at $\kappa=\infty$ that the universality class of the random geometry changes.

Physically then, the flat lattice system at $\kappa=\infty$ is entropically unstable to the formation of baby universes that make up the full gravitational ensemble, which gives a worldsheet geometric picture of why there is no flattening phase transition in the twodimensional $R^{2}$ quantum gravity model. In the remainder of this paper we will deduce what the nature is of the phase when one perturbs the flat graph ensemble by baby universe outgrowths as described above. To get an idea of what the system at $\kappa<\infty$ represents, consider the simplification whereby we allow outgrowths of only a single loop side $\ell$. This means that we keep only the $n=\ell$ term in (4.1) and proceeding as in the general case we would then arrive at

$$
\widehat{\mathcal{Z}}_{\ell}(\mu, \kappa)=\widehat{\mathcal{Z}}_{\ell}^{(0)}\left(\bar{\mu}_{\ell}\right)
$$

This is the standard behaviour of the generating function for a branched polymer ensemble [11. Indeed, as before, $\widehat{\mathcal{Z}}_{\ell}(\mu, \kappa)$ cannot diverge at the critical point $\mu_{c}$ because then $\bar{\mu}_{\ell}(\mu, \kappa)$ would reach 0 before $\widehat{\mathcal{Z}}_{\ell}(\mu, \kappa)$ reaches $\infty$. Differentiating both sides of (4.8) with respect to $\mu$ using (4.5) yields

$$
\frac{\partial \widehat{\mathcal{Z}}_{\ell}(\mu, \kappa)}{\partial \mu}=\frac{\left(1-3 \ell^{2} \mathrm{e}^{\mu \ell^{2}-\kappa w(\ell)} \widehat{\mathcal{Z}}_{\ell}(\mu, \kappa)\right) \mathrm{e}^{-4 \ell^{2}\left(\mu-\bar{\mu}_{\ell}\right)} \frac{\partial \widehat{\mathcal{Z}}_{\ell}^{(0)}\left(\bar{\mu}_{\ell}\right)}{\partial \bar{\mu}_{\ell}}}{1+\mathrm{e}^{-\ell^{2}\left(3 \mu-4 \bar{\mu}_{\ell}\right)-\kappa w(\ell)} \frac{\partial \widehat{\mathcal{Z}}_{\ell}^{(0)}\left(\bar{\mu}_{\ell}\right)}{\partial \bar{\mu}_{\ell}}} .
$$

Since $\partial \widehat{\mathcal{Z}}_{\ell}^{(0)}\left(\bar{\mu}_{\ell}\right) / \partial \bar{\mu}_{\ell} \rightarrow-\infty$ as $\bar{\mu}_{\ell} \rightarrow 0$, it follows that the denominator of (4.9) vanishes at some finite value of $\bar{\mu}_{\ell}$ where the numerator is regular and non-vanishing. Thus $\partial \widehat{\mathcal{Z}}_{\ell}(\mu, \kappa) / \partial \mu$ diverges at the critical point, and generically we get a branched polymer ensemble with string susceptibility exponent $\gamma_{\text {str }}=+\frac{1}{2}$ [1]. However, the actual bottleneck ensemble contains a complicated mixing of all loop lengths and more care must be exercised in deducing the critical behaviour.

We begin by deducing some analytic properties of $\widehat{\mathcal{Z}}_{\ell}(\mu, \kappa)$ for $\kappa<\infty$ using the recursion relation (4.3). Note that for fixed $\ell, \widehat{\mathcal{Z}}_{\ell}(\mu, \kappa)$ is a monotonic decreasing function of $\mu \in \mathbb{R}^{+}$, while for fixed $\mu$ it is a decreasing function of $\ell$. Let us examine the behaviour of the functions $\bar{\mu}_{n}$ very close to the critical point $\mu=\mu_{c}$. Since $\mu_{c}=0$ at $\kappa=\infty$, at very large $\kappa$ we expect that $\mu_{c}$ is very small so that for $n^{2} \mu \ll 1$ we find

$$
\begin{aligned}
\bar{\mu}_{n+1}-\bar{\mu}_{n}> & \frac{1}{4 n^{2}} \log \left(1+4 n^{2} \mathrm{e}^{\mu n^{2}-\kappa w(n)} \widehat{\mathcal{Z}}_{n}(\mu, \kappa)\right) \\
& -\frac{1}{4(n+1)^{2}} \log \left(1+4(n+1)^{2} \mathrm{e}^{\mu(n+1)^{2}-\kappa w(n+1)} \widehat{\mathcal{Z}}_{n}(\mu, \kappa)\right) \\
\simeq & \frac{1}{4 n^{2}} \log \left(1+4 n^{2} \mathrm{e}^{-\kappa w(n)} \widehat{\mathcal{Z}}_{n}\left(\mu_{c}, \kappa\right)\right) \\
& -\frac{1}{4(n+1)^{2}} \log \left(1+4(n+1)^{2} \mathrm{e}^{-\kappa w(n+1)} \widehat{\mathcal{Z}}_{n}\left(\mu_{c}, \kappa\right)\right) \\
> & 0
\end{aligned}
$$


for $\mu \sim \mu_{c}$ and $\kappa \gg 1$. Thus $\left\{\bar{\mu}_{n}\right\}$ is an increasing sequence for small $n$. Denoting $\bar{\mu}_{\text {min }}=\inf _{n} \bar{\mu}_{n}$, it follows from (4.2) and (4.6) that

$$
\widehat{\mathcal{Z}}_{\ell}(\mu, \kappa) \leq \mathrm{e}^{-\bar{\mu}_{\min } \ell^{2}} g_{1}\left(\bar{\mu}_{\min }\right)\left(\ell+\frac{1}{2} \sqrt{\frac{\pi}{\bar{\mu}_{\min }}}\right) .
$$

On the other hand, because $\bar{\mu}_{\text {min }}<\mu$, from (4.3) it follows that for large $\ell$ we have

$$
\widehat{\mathcal{Z}}_{\ell}(\mu, \kappa)>\mathrm{e}^{-\bar{\mu}_{\min } \ell^{2}}
$$

The bounds (4.11) and (4.12) show that for large $\ell$ we may write

$$
\widehat{\mathcal{Z}}_{\ell}(\mu, \kappa) \simeq \mathrm{e}^{-\bar{\mu}_{\min } \ell^{2}} f(\ell, \mu)
$$

where the function $f(\ell, \mu)$ is larger than 1 and grows at most linearly with $\ell$. We then see that at large enough $n$

$$
\bar{\mu}_{n+1}-\bar{\mu}_{n} \simeq \frac{\log 4 n^{2}}{4 n^{2}}-\frac{\log 4(n+1)^{2}}{4(n+1)^{2}}+\frac{\kappa w(n+1)}{4(n+1)^{2}}-\frac{\kappa w(n)}{4 n^{2}}
$$

so that now, depending upon the nature of the function $w(n)$, the $\bar{\mu}_{n}$ may start to fall again. However, we also have that

$$
\bar{\mu}_{\infty} \equiv \lim _{n \rightarrow \infty} \bar{\mu}_{n}=\frac{3}{4} \mu+\frac{1}{4} \bar{\mu}_{\min }>\bar{\mu}_{\min }
$$

so that we can identify $\bar{\mu}_{\min }=\bar{\mu}_{1}$. It follows from these properties of the sequence $\left\{\bar{\mu}_{n}\right\}$ that the infinite series in (4.3) is absolutely convergent.

We now rewrite (4.3) in the form

$$
\widehat{\mathcal{Z}}_{\ell}(\mu, \kappa)=\widehat{\mathcal{Z}}_{1}(\mu, \kappa)+\widehat{\mathcal{H}}_{\ell}^{(0)}(\mu)-\widehat{\mathcal{H}}_{1}^{(0)}(\mu)+\sum_{n=1}^{\ell-1}\left(\widehat{\mathcal{Z}}_{\ell}^{(0)}\left(\bar{\mu}_{n}\right)-\widehat{\mathcal{Z}}_{n}^{(0)}\left(\bar{\mu}_{n}\right)\right)
$$

The right-hand side of (4.16) depends only on the known functions $\widehat{\mathcal{H}}_{\ell}^{(0)}(\mu)$ and the partition functions $\widehat{\mathcal{Z}}_{n}(\mu, \kappa)$ for $1 \leq n<\ell$. Iterating (4.16) thus determines $\widehat{\mathcal{Z}}_{n}(\mu, \kappa)$ as a function only of $\mu, \kappa$, and $\widehat{\mathcal{Z}}_{1}$, for any $n>1$. Using (4.3) we see that $\widehat{\mathcal{Z}}_{1}$ satisfies

$$
\widehat{\mathcal{Z}}_{1}(\mu, \kappa)=\widehat{\mathcal{H}}_{1}^{(0)}(\mu)+\mathcal{F}\left(\mu, \kappa, \widehat{\mathcal{Z}}_{1}(\mu, \kappa)\right)
$$

where implicitly the function $\mathcal{F}$ is given by

$$
\begin{aligned}
\mathcal{F}\left(\mu, \kappa, \widehat{\mathcal{Z}}_{1}\right) & =\sum_{n=1}^{\infty} \widehat{\mathcal{Z}}_{n}^{(0)}\left(\bar{\mu}_{n}\right) \\
\bar{\mu}_{n}\left(\mu, \kappa, \widehat{\mathcal{Z}}_{n}\right) & =\mu-\frac{1}{4 n^{2}} \log \left(1+4 n^{2} \mathrm{e}^{\mu n^{2}-\kappa w(n)} \widehat{\mathcal{Z}}_{n}\right)
\end{aligned}
$$

together with (4.16), but explicitly it is a very complicated function. Moreover, (4.19) is an analytic function of $\mu, \kappa$, and $\widehat{\mathcal{Z}}_{1}$. 
Differentiating (4.17) with respect to $\mu$ gives

$$
\left.\frac{\partial \widehat{\mathcal{Z}}_{1}}{\partial \mu}\right)_{\kappa}=\frac{\left.\frac{d \widehat{\mathcal{H}}_{1}^{(0)}}{d \mu}+\frac{\partial \mathcal{F}}{\partial \mu}\right)_{\kappa, \widehat{\mathcal{Z}}_{1}}}{\left.1-\frac{\partial \mathcal{F}}{\partial \widehat{\mathcal{Z}}_{1}}\right)_{\mu, \kappa}}
$$

The numerator of $(4.20)$ is a well behaved function for $\mu>0$ but at some critical point the denominator will vanish and thereby generate a non-analyticity in $\widehat{\mathcal{Z}}_{1}$. To see this, we note from (4.19) that

$$
\left.\frac{\partial \mathcal{F}}{\partial \widehat{\mathcal{Z}}_{1}}\right)_{\mu, \kappa}=\sum_{n=1}^{\infty} \mathcal{A}^{n, n} f_{n}
$$

where we define

$$
\mathcal{A}^{\ell, n}=-\widehat{\mathcal{Z}}_{\ell}^{(0) \prime}\left(\bar{\mu}_{n}\right) \mathrm{e}^{n^{2}\left(4 \bar{\mu}_{n}-3 \mu\right)-\kappa w(n)}
$$

and

$$
\left.f_{\ell} \equiv \frac{\partial \widehat{\mathcal{Z}}_{\ell}}{\partial \widehat{\mathcal{Z}}_{1}}\right)_{\mu, \kappa}=1+\sum_{n=1}^{\ell-1}\left(\mathcal{A}^{\ell, n}-\mathcal{A}^{n, n}\right) f_{n}
$$

where we have used (4.16). Note that $\mathcal{A}^{\ell, n}$ is a monotonically decreasing function of $\ell$, so that (4.23) implies

$$
f_{\ell+1}-f_{\ell}=\sum_{n=1}^{\ell}\left(\mathcal{A}^{\ell+1, n}-\mathcal{A}^{\ell, n}\right) f_{n}<0
$$

provided that $f_{n}>0 \forall n \geq 1$. Thus $\left\{f_{\ell}\right\}$ is a monotonically decreasing sequence. Under these same assumptions we also have

$$
\begin{aligned}
\sum_{n=1}^{\ell-1} \mathcal{A}^{\ell, n} f_{n} & =\sum_{n=1}^{\ell-1} f_{n}\left(\ell^{2} g_{\ell}\left(\bar{\mu}_{n}\right)-g_{\ell}^{\prime}\left(\bar{\mu}_{n}\right)\right) \mathrm{e}^{-3 n^{2}\left(\mu-\bar{\mu}_{n}\right)-\kappa w(n)} \mathrm{e}^{\left(n^{2}-\ell^{2}\right) \bar{\mu}_{n}} \\
& <\mathrm{e}^{-(2 \ell-1) \bar{\mu}_{1}} \sum_{n=1}^{\ell-1} f_{n}\left(\ell^{2} g_{\ell}\left(\bar{\mu}_{n}\right)-g_{\ell}^{\prime}\left(\bar{\mu}_{n}\right)\right) \mathrm{e}^{-3 n^{2}\left(\mu-\bar{\mu}_{n}\right)-\kappa w(n)} .
\end{aligned}
$$

The last line of (4.25) vanishes in the limit $\ell \rightarrow \infty$, so that the first series in the recursion relation (4.23) vanishes as $\ell \rightarrow \infty$. Using this fact and (4.23) it follows that (4.21) can be written in terms of the single quantity $f_{\infty}=\lim _{\ell \rightarrow \infty} f_{\ell}$ as

$$
\frac{\partial \mathcal{F}}{\partial \widehat{\mathcal{Z}}_{1}}=1-f_{\infty}
$$

Again assuming that the sequence $\left\{f_{\ell}\right\}$ is monotonically decreasing we have that the limit of the sequence has the lower bound

$$
f_{\infty}>1-\sum_{n=1}^{\infty} \mathcal{A}^{n, n}
$$

which is positive at large enough $\mu$. On the other hand, as $\mu$ decreases the sequence will go negative and then start to oscillate. It is clear that at small enough $\mu$ this must happen because it is straightforward to choose a $\mu$ for which $f_{2}$ is already negative. It follows 
by continuity that the critical point where the sequence $\left\{f_{\ell}\right\}$ monotonically decreases to $f_{\infty}=0$ must exist, and therefore the system is a branched polymer.

To determine the value of $\gamma_{\text {str }}$ we consider two cases. If $w(n)$ is a strictly increasing function of $n$, then by working at very large $\kappa$ we can ensure that the first term in (4.21) dominates so that at the critical point

$$
1 \simeq\left(g_{1}\left(\bar{\mu}_{1}\right)-g_{1}^{\prime}\left(\bar{\mu}_{1}\right)\right) \mathrm{e}^{-\kappa}
$$

and higher terms are suppressed by factors of $\mathrm{e}^{-\kappa(w(n)-w(1))}$. As long as $\left\{f_{\ell}\right\}$ is a decreasing sequence, the sum of terms with $n \geq 2$ in (4.21) converges to something negligible compared to the first term. Now we can make the standard argument that the denominator of 4.20$)$ will vanish linearly with $\widehat{\mathcal{Z}}_{1}(\mu, \kappa)$ which we assume to behave as

$$
\widehat{\mathcal{Z}}_{1}(\mu, \kappa)=\widehat{\mathcal{Z}}_{1}\left(\mu_{c}(\kappa), \kappa\right)-B\left(\mu_{c}(\kappa), \kappa\right)\left(\mu-\mu_{c}(\kappa)\right)^{1-\gamma_{\text {str }}}
$$

where the function $B$ is regular at the critical point, and therefore $\gamma_{\text {str }}=\frac{1}{2}$. In the case that $w(n)$ is constant this argument fails. To show that the model has generic branched polymer behaviour we would need to show that

$$
\left.\frac{\partial^{2} \mathcal{F}}{\partial \widehat{\mathcal{Z}}_{1}^{2}}\right)_{\mu, \kappa} \neq 0 \quad \text { when } \quad f_{\infty}=0
$$

but we have not succeeded in finding a proof of this. However, it is straightforward to solve the recursion equations (4.23) numerically and fig. 3 shows the behaviour of $f_{\infty}$ for $\mathrm{e}^{-\kappa}=10^{-4}$. It is clear that $\gamma_{\mathrm{str}}=\frac{1}{2}$ in this case as well.

We conclude that the system defines a generic branched polymer phase of two dimensional quantum gravity with string susceptibility exponent $\frac{1}{2}$ as soon as the coupling $\kappa$ is tuned away from infinity. This model only allows a rather simple subset of all possible branchings and we expect that the ordinary pure gravity phase (with $\gamma_{\text {str }}=-\frac{1}{2}$ ) is recovered by including all of the more complicated branchings in which the path on the base graph is allowed to be arbitrary. The basic point is that the model above gives a physical understanding for the absence of a flat phase when the curvature coupling constant $\kappa$ is large.

This work was supported in part by PPARC grant \# GR/L56565 and the Danish Natural Science Research Council. 


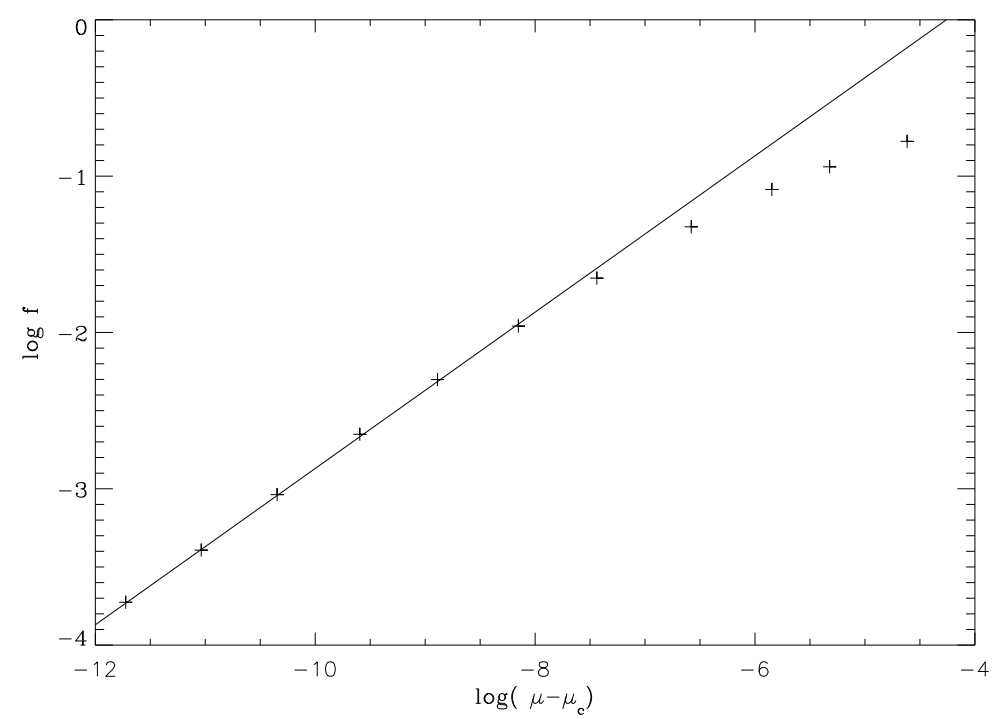

Figure 3: Logarithmic plot of $f_{\infty}$ against $\mu$ for the case $w(n)=1, \mathrm{e}^{-\kappa}=10^{-4}$. The crosses represent computed values with $\mu_{c}=0.040112$ and the straight line has slope $\frac{1}{2}$.

\section{References}

[1] F. David, in: Gravitation and Quantization, Les Houches Session LVII (1992);

J. Ambjørn, in: Fluctuating Geometries and Statistical Mechanics, Les Houches Session LXII (1994);

P. Di Francesco, P. Ginsparg and J. Zinn-Justin, Phys. Rep. 254 (1995) 1;

J. Ambjørn, B. Durhuus and T. Jónsson, Quantum Geometry (Cambridge University Press, 1997).

[2] H. Kawai and R. Nakayama, Phys. Lett. B 306 (1993) 224;

S. Ichinose, Nucl. Phys. B 445 (1995) 311.

[3] M.J. Bowick, S.M. Catterall and G. Thorleifsson, Phys. Lett. B 391 (1997) 305.

[4] V.A. Kazakov, M. Staudacher and T. Wynter, Nucl. Phys. B 471 (1996) 309.

[5] N. Ferguson and J.F. Wheater, Phys. Lett. B 319 (1993) 104.

[6] J.D. Correia and J.F. Wheater, Phys. Lett. B 388 (1996) 707.

[7] S. Jain and S.D. Mathur, Phys. Lett. B 286 (1992) 239;

J. Ambjørn, S. Jain and G. Thorleifsson, Phys. Lett. B 297 (1993) 34;

J. Ambjørn and G. Thorleifsson, Phys. Lett. B 323 (1994) 7.

[8] B. Li, N. Madras and A.D. Sokal, J. Stat. Phys. 80 (1995) 661.

[9] G. Moore, N. Seiberg and M. Staudacher, Nucl. Phys. B 362 (1991) 665.

[10] J. Ambjørn, B. Durhuus, J. Fröhlich and P. Orland, Nucl. Phys. B 270 [FS16] (1986) 457 ;

J. Ambjørn, B. Durhuus and T. Jónsson, Phys. Lett. B 244 (1990) 403;

P. Bialas and Z. Burda, Phys. Lett. B 384 (1996) 75.

[11] B. Durhuus, Nucl. Phys. B 426 (1994) 203. 